\title{
Reduced GABA content in the motor thalamus during effective deep brain stimulation of the subthalamic nucleus
}

\section{Alessandro Stefani ${ }^{1,2 *}$, Ernesto Fedele ${ }^{3,4}$, Mariangela Pierantozzi ${ }^{2}$, Salvatore Galati ${ }^{5}$, Francesco Marzetti ${ }^{2}$, Antonella Peppe ${ }^{1}$, Francesco Saverio Pastore ${ }^{2}$, Giorgio Bernardi ${ }^{1,2}$ and Paolo Stanzione ${ }^{1,2}$}

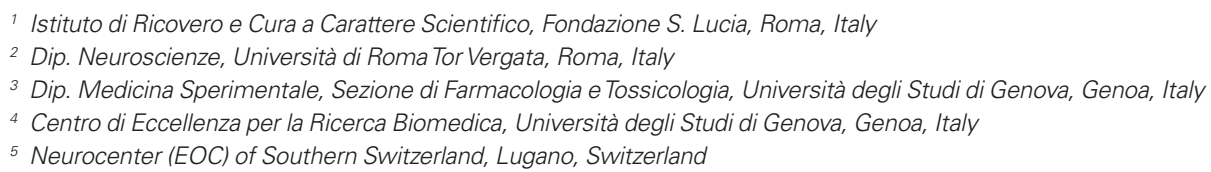

\section{Edited by:}

Jose Bargas, National Autonomous

University of Mexico, Mexico

\section{Reviewed by:}

Gordon Arbuthnott, Okinawa Institute of Science and Technology, Japan William Hutchison, University Health Network, Canada

*Correspondence:

Alessandro Stefani, Dip Neuroscienze Univ Tor Vergata, V.le Montpellier, 1 00133 Rome, Italy.

e-mail:stefani@uniroma2.it
Deep brain stimulation (DBS) of the subthalamic nucleus (STN), in Parkinson's disease (PD) patients, is a well established therapeutic option, but its mechanisms of action are only partially known. In our previous study, the clinical transitions from OFF- to ON-state were not correlated with significant changes of GABA content inside GPi or substantia nigra reticulata. Here, biochemical effects of STN-DBS have been assessed in putamen (PUT), internal pallidus (GPi), and inside the antero-ventral thalamus (VA), the key station receiving pallidothalamic fibers. In 10 advanced PD patients undergoing surgery, microdialysis samples were collected before and during STN-DBS. cGMP, an index of glutamatergic transmission, was measured in GPi and PUT by radioimmunoassay, whereas GABA from VA was measured by HPLC. During clinically effective STN-DBS, we found a significant decrease in GABA extracellular concentrations in VA $(-30 \%)$. Simultaneously, cGMP extracellular concentrations were enhanced in PUT (+200\%) and GPi (+481\%). These findings support a thalamic dis-inhibition, in turn re-establishing a more physiological corticostriatal transmission, as the source of motor improvement. They indirectly confirm the relevance of patterning (instead of mere changes of excitability) and suggest that a rigid interpretation of the standard model, at least when it indicates the hyperactive indirect pathway as key feature of hypokinetic signs, is unlikely to be correct. Finally, given the demonstration of a key role of VA in inducing clinical relief, locally administration of drugs modulating GABA transmission in thalamic nuclei could become an innovative therapeutic strategy.

Keywords: stereotactic neurosurgery, subthalamus, globus pallidus, antero-ventral thalamus, GABA, glutamate, cGMP, UPDRS

\section{INTRODUCTION}

In early 2000s, it was proposed, asides from the traditional neurosurgery performed on a single target for severe Parkinson's disease (PD) patients (commonly the subthalamic nucleus - STN - or the globus pallidus pars interna-GPi) the so-called "multi-target strategy," consisting of simultaneous and bilateral implantation of multiple basal ganglia (BG) nuclei (Peppe et al., 2004; Stefani et al., 2009). In principle, our genuine purpose was to improve treatment of specific signs of clinical Parkinsonism, for example ensuring a more efficient control of involuntary movements. With this goal in mind, in a cohort of advanced PD patients afflicted by rather disabling dyskinesias, both STN and GPi were implanted bilaterally. As a consequence, it was possible to conclude that GPiDBS provided efficacious recovery from hypokinesia that was not significantly worse than STN-DBS (Peppe et al., 2004), years before recent comparative investigations (Follett et al., 2010).

However, the simultaneous switch-ON of both targets did not produce higher benefits than each target alone, neither on dyskinesia nor in the management of axial signs. Rather, the clinical indication for that sort of invasive - and costly - "double-targeting" approach tended to become less in time.
Nevertheless, that research line allowed us to study biochemical changes occurring, simultaneously, in GPi as well as in the ventral anterior thalamus (VA), the crucial structure conveying pallidofugal information to cortex, and in putamen (PUT). Indeed, it was possible to measure endogenous amino acids, collectible from extracellular space intra-GPi, intra-VA, and intra-PUT, before and during an efficacious DBS delivery (Fedele et al., 2001; Stefani et al., 2005, 2006; Galati et al., 2006).

At first, at odd with traditional views, the administration of the dopamine agonist apomorphine, although clinically efficacious, failed to modify GABA and glutamate content in GPi. Also 30-40 min STN-DBS (with clinically relevant parameters) barely affected GABA, taurine, aspartate, and glutamate concentrations (Fedele et al., 2001). On the other hand, STN-DBS had promoted the augmentation of the GPi [and substantia nigra pars reticulata (SNr)] cGMP levels (Stefani et al., 2005; Galati et al., 2006). The implicit interpretation of these unexpected findings was that, instead of silencing STN-mediated transmission, DBS might dictate a clustered and increased discharge pattern in SNr. Those data were initially considered paradoxical, since in conflict, at least in part, with findings collected in rodent's disease model. For instance, some 
rat studies showed the prevalent inhibition of SNr during STNDBS (Windels et al., 2003, 2005). These inconsistencies may reveal that the abrupt dopamine depletion by 6-OHDA, possibly for the overwhelming role of fast occurring compensatory mechanisms, is not fit to investigate properly the electrophysiological and chemical features that PD imposes.

If we consider the cGMP increase as an indirect estimation of neuronal activity in GPi during STN-DBS (Fedele and Raiteri, 1999), our findings were in theoretical accord with the STN-DBSmediated excitation and clustering of the GPi firing pattern as observed in MPTP primates (Hashimoto et al., 2003).

Yet, the relevance of the cGMP finding, per se, was uncertain; after all, it depends on an acute change of the NMDA-NO endogenous transmission (notoriously impaired in extrapyramidal disorders, but not exactly a routine therapeutic target!).

Is it feasible that similar changes in cGMP levels are really correlated with consistent clinical amelioration in every PD patient?

How could we further deepen our knowledge on the biochemical counterparts of the OFF-ON behavioral transitions?

Do the observed changes in the first output stations (GPi/SNr) affect the motor thalamus, the crucial structure conveying motor information to cortex?

How STN-DBS influence intra thalamic GABA and, in turn, corticostriatal transmission?

Therefore, we investigated the extracellular GABA concentration in VA during the first delivery of STN-DBS $(n=10)$, at clinically relevant or null voltage. Simultaneously, in the same PD patients, the cGMP level was measured in GPi $(n=10)$ and PUT $(n=6)$.

In synthesis, our results will show that DBS reversibly reduces $(-30 \%)$ VA GABA levels, increases cGMP levels in GPi and also significantly increases cGMP concentration in PUT, as far as the clinical transition to $\mathrm{ON}$-state takes place.

Our findings sound in accord with the growing body of evidence refreshing and reforming the standard model of BG circuitry. The dogma about an hyperactive indirect pathway as a crucial hallmark of Parkinsonian hypokinetic signs, for instance, has been revisited since the relief from akinesia and rigidity occurs in the absence of reduced GPi excitability and changes of GPi GABA levels. However, clinical "ON-state" requires a rapid change in thalamic GABA content, as here described. Therefore, these results confirm, in line with older models, that VA is the core clinical player in determining thalamocortical transmission.

\section{PATIENTS AND METHODS}

Ten advanced $P D$ patients (mean age $=57.6 \pm 9.2$ years; mean UPDRS score in $\mathrm{OFF}=47.7 \pm 8.1$; mean disease duration $=11 \pm 3.1$ years) were included in this study according to selection criteria previously reported (Fedele et al., 2001; Stefani et al., 2006). Stimulating permanent electrodes were implanted in both the STN and the GPi, bilaterally. For trajectory details, and implantation procedures, see Figure 1 legend and References (Fedele et al., 2001; Stefani et al., 2005, 2006). Written, informed consent was obtained from each patient. The Local Ethics Committee approved the protocol and consent form describing the risks and potential benefits of the study. At time for surgery, every patient was under washout (at least overnight withdrawal from L-DOPA and 3-day suspension of dopamine-agonists).
Probes were infused with sterile PBS ( $5 \mu \mathrm{L} / \mathrm{min}$ ) for stabilization (90 min). Then, basal microdialysis samples (50 $\mu \mathrm{L}$ each) and clinical data were collected for $50 \mathrm{~min}$. Afterward, STN-DBS was switched on for $60 \min (n=10$ patients, Table 1 and Figure 2). Stimulation parameters were: $90 \mu \mathrm{s}, 130 \mathrm{~Hz}$ and mean $2.4 \mathrm{~V}$ (but see Figure 1 legend). After $60 \mathrm{~min}$ DBS, $60 \mathrm{~min}$ of recovery were performed. All procedures were without additional risks for the patient, since guide tubes for micro-recordings were already placed in each target (STN and GPi).

Clinical changes were assessed utilizing selected items of UPDRS (rigidity $0-4$, finger tapping $0-4$, hand movement $0-4$; total $=0$ corresponds to "normal," 12 maximum score) by a neurologist blind to the stimulus intensity between 0 and $3 \mathrm{~V}$. GABA and cGMP were determined by HPLC and by RIA, respectively.

Significance of GABA and cGMP changes was assessed by comparing the mean of the concentrations obtained in the single fractions under basal conditions with those during STN-DBS and during recovery conditions. Mean values were compared by ANOVA two way (first main factor: treatment with three levels: basal, treatment, recovery; second main factor: time with six levels) corrected by Greenhouse-Geisser correction and followed by post hoc Scheffè test to assess the significance of the differences between single fractions. Given the not demonstrated normality of biochemical data the results were also confirmed by nonparametric Friedman ANOVA followed by Wilcoxon as post hoc test. Clinical scores were compared using similar non-parametric methods. Correlation were studied between the maximal changes induced by DBS in the GABA and cGMP concentrations (Pearson parametric correlations) and clinical score (non-parametric Spearman correlations). The changes were evaluated as the maximum change of each of these parameters in comparison to their mean basal score.

\section{RESULTS}

\section{CLINICAL EFFECT OF DBS}

For all the fore-coming biochemical results during STN-DBS, consider that they correlate with a significant change of UPDRS (at least 30\% amelioration of main examined motor features). To note, post hoc Wilcoxon test showed a significant UPDRS score amelioration from the first $10 \mathrm{~min}$ of DBS in comparison to all the basal score. Only in case of PUT cGMP, the statistical data comparing biochemical and clinical scores did not reach significance (but because of the small sample).

\section{GPi cGMP}

Deep brain stimulation produced a clear cut increase of cGMP levels (Table 1, Figure 2; average $+606.2 \%$, ANOVA main effect $\mathrm{df}=2 / 18 ; \varepsilon=0.515 ; \mathrm{df}=1.03 / 9.28 ; F=20.56, p=0.00002)$. Post hoc Scheffé test showed: basal vs DBS $p=0.000047$; DBS vs recovery $p=0.000489$. Friedman ANOVA followed by Wilcoxon confirmed these significances.

To note, the quantification of intra-GPi GABA, in this series of patients, was not performed since already investigated in our seminal paper (Fedele et al., 2001); to mention, no significant change of GPi GABA was observed even following prolonged and clinically effective stimulation periods. 


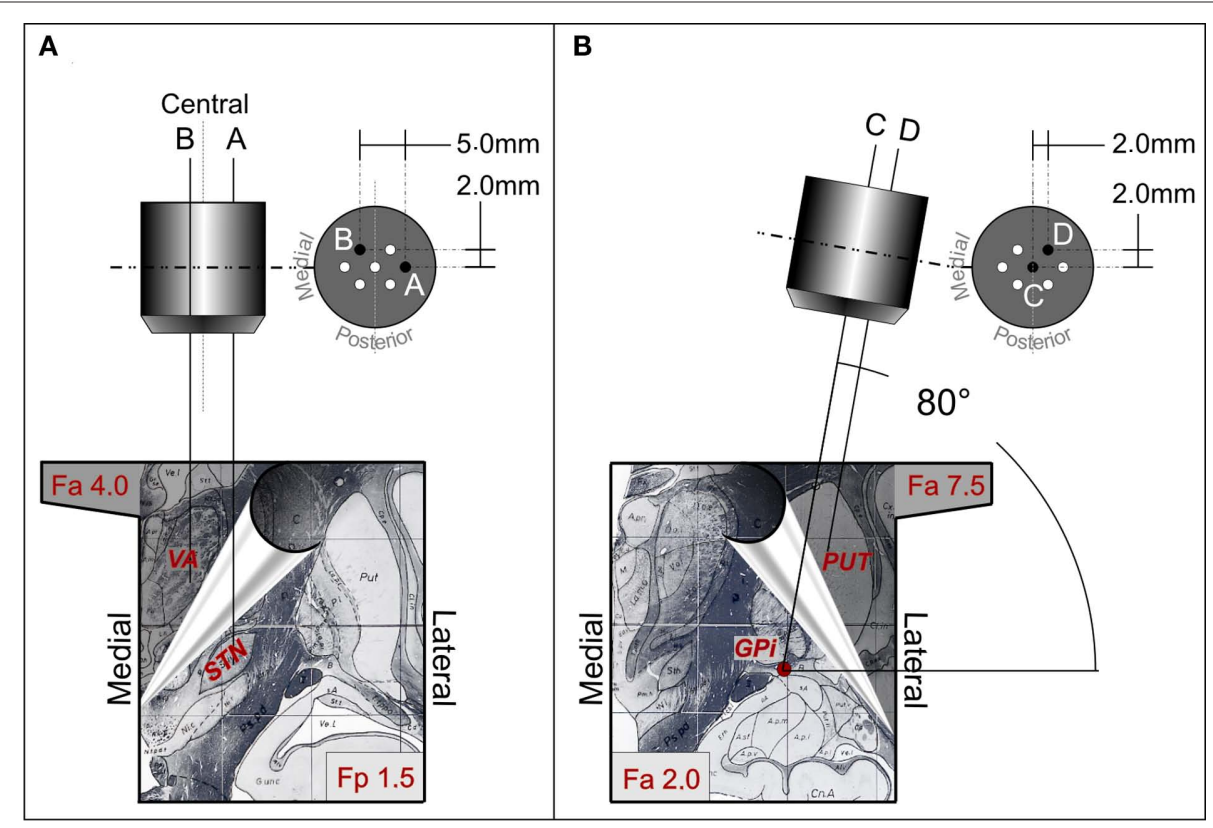

FIGURE 1 |Trajectories of the multi-electrodes-probes holders aimed at the STN-VA (A) and at the PUT-GPi (B). Each section shows representative diagrams of the utilized multi-electrode holder (top) and, at the bottom, two different partially superimposed sections of atlas (note the hand leafing the superior section). The left bottom panel shows coronal atlas section at $1.5 \mathrm{~mm}$ posterior to the inter-commissural midline (Fp 1.5, STN target) and at $4 \mathrm{~mm}$ anterior to the inter-commissural midline (Fa 4.0 VA target). The right bottom panel illustrates coronal atlas section at $2 \mathrm{~mm}$ anterior to the inter-commissural midline (Fa 2.0 GPi target) and at $7.5 \mathrm{~mm}$ anterior to the inter-commissural midline (Fa 7.5 PUT target). To reach STN passing through the VA, the central electrode of the multi-electrode holder (A) was aimed at a target at $8.5 \mathrm{~mm}$ of laterality, $-4 \mathrm{~mm}$ with respect to the AC-PC plane, and at the mid point of the AC-PC line. The trajectory had an angle of $70^{\circ}-75^{\circ}$ in sagittal plane and of $85^{\circ}-90^{\circ}$ in the coronal plane. The more lateral electrode of the multi-electrode holder [electrode $A$ in (A)] was at $3.5 \mathrm{~mm}$ of laterality from the central one. This laterality allowed to reach a target at $12.0 \mathrm{~mm}$ of laterality from the mid line, which is the usual location of the STN target. On the contrary, the electrode B trajectory was $1.5 \mathrm{~mm}$ more medial than the central one and $2 \mathrm{~mm}$ more anterior. The probe membrane ( $4 \mathrm{~mm}$ length) was positioned between 11.0 and $7.0 \mathrm{~mm}$ above the target ( +7 to $+3 \mathrm{~mm}$ above the inter-commissural plane), along the trajectory of electrode B. Given the angle in the sagittal planes $\left(70^{\circ}-75^{\circ}\right)$, the trajectory had a laterality of $7.0 \mathrm{~mm}$ and, at the level of +7 to $+3 \mathrm{~mm}$ above the inter-commissural plane, its antero-posterior position was between +6.0 and $+4.0 \mathrm{~mm}$ with respect to the midpoint of the inter-commissural line, corresponding to the center of VA. After electrophysiological identification of the targets, the recording electrode in the GPi was replaced by a microdialysis probe [trajectory $C$ in $(\mathbf{B})$ ]. A second probe was positioned in trajectory $D$, at the level of PUT [D in (B)], utilizing the antero-lateral hole of the multi-electrode holder according to the coronal angle used. Probe membrane was positioned at a laterality between 22.5 and $25.0 \mathrm{~mm}$, a depth of +9.0 with respect to the inter-commissural plane, and between +4.8 and $+7.5 \mathrm{~mm}$ anterior to the midpoint of the inter-commissural line. Probes and stimulating electrode real positions were verified during surgery by $\mathrm{X}$-ray in antero-posterior and latero-lateral projection.

\section{MOTOR THALAMUS GABA}

STN-DBS produced a significant decrease in GABA level during stimulation (Table 1, Figure 2; average-31.1\%; ANOVA main effect $\mathrm{df}=2 / 18 ; \varepsilon=0.784 ; \mathrm{df}=1.56 / 14.11, F=11.83, p=0.000523 ; 0.0016$ Greenhouse-Geisser correction). Post hoc Scheffé test showed: basal vs DBS $p=0.000783$; DBS vs recovery $p=0.00413$. Friedman ANOVA followed by Wilcoxon confirmed these levels of significance.

\section{PUTAMEN cGMP}

DBS produced a clear cut increase of PUT cGMP $(n=6)$, as already partially described (Stefani et al., 2006). The average increase was $220 \%$; the mean value was significantly higher (Wilcoxon test, $Z=2.52$, $p<0.05)$ than the mean basal and recovery. In each patient, the rise of PUT cGMP was clear from the section following DBS-ON (Figure 2).

\section{DISCUSSION}

In the classical BG model (Albin et al., 1989; DeLong, 1990), PD akinesia results from an imbalance between indirect and direct pathways and correlates with an overactivity of the STN and the GPi.
Since the early nineties, the similar efficacy promoted by STN-DBS and subthalamotomy suggested that high-frequency stimulation acted through a sort of functional ablation. The so-called "suppression hypothesis" (Benazzouz et al., 2000; Filali et al., 2004) insisted on firing silencing as the major DBS mechanism of action. Whilst such hypothesis gained credit inside STN (Filali et al., 2004), since each stimulus was in fact followed by a no-spiking time window, it was abundantly challenged by evidence collected in target stations (GPi/SNr) in primates and humans (see later).

To further complicate this issue, studies performed in rodent's models of PD have shown quite conflicting data. Some groups have described a prevalent inhibition of SNr during STN-DBS, through GABA release eventually from GP, utilizing stimulation period abundantly sufficient to induce behavioral changes (up to 1 h, Windels et al., 2003, 2005). Others, by means of multi-channel recordings in freely moving rats described the prevalence of a mixed response ("nearly equal numbers of excitatory and inhibitory responses were found in the GP and $\mathrm{SNr}$ ") and a relevant decrease of burst firing (Shi et al., 2006). 


\begin{tabular}{|c|c|c|c|}
\hline & Basal & STN-DBS & Recovery \\
\hline \multicolumn{4}{|c|}{ cGMP-GPi (fmol/50 $\mu \mathrm{L}, n=10)$} \\
\hline Mean & 1.6 & 9.7 & 3.1 \\
\hline SD & 0.5 & 5.7 & 1.6 \\
\hline \multicolumn{4}{|c|}{ GABA-VA (pmol/50 $\mu \mathrm{L}, n=6)$} \\
\hline Mean & 4.5 & 3.1 & 4.2 \\
\hline SD & 0.5 & 0.8 & 0.9 \\
\hline \multicolumn{4}{|c|}{ cGMP-PUT (fmol/50 $\mu \mathrm{L}, n=6$ ) } \\
\hline Mean & 1.45 & 3.2 & 1.8 \\
\hline SD & 0.1 & 1.3 & 0.3 \\
\hline \multicolumn{4}{|c|}{ UPDRS section III (selected items) } \\
\hline Mean & 8.4 & 5.5 & 8.2 \\
\hline SD & 1.0 & 1.4 & 0.9 \\
\hline
\end{tabular}

In humans and non-human primates (NHP), we and others have documented that the clinical benefit may parallel an increased - and not decreased-GPi and SNr discharge rate and, more importantly, a dramatic modulation of their pattern of activity (Hashimoto et al., 2003; Galati et al., 2006). Accordingly, as detailed since our pioneering papers in the GPi of PD patients, DBS failed to affect amino acids levels (no GABA decrease, in particular, Fedele et al., 2001) but caused a large increment of the cGMP title (Stefani et al., 2005).

Whereas the negligible modification of glutamate could be attributable to lack of sensitivity of the technique, the lack of GABA concentration change suggests that a strong dampening of excitability (in $\mathrm{GPi} / \mathrm{SNr}$ ) is not likely to occur during clinically relevant STN-DBS (whilst it is the rule under apomorphine! i.e., Stefani et al., 1997, 1999).

Investigations performed in MPTP-treated primates sound more in agreement with our central hypotheses. The GPi neuronal activity recorded during efficacious STN-DBS is indeed more regular, being time locked to the stimulus pulse, and had less bursting activity (Hashimoto et al., 2003; Hahn et al., 2008).

The mismatch between 6-OHDA rodents and humans is puzzling but highlights once more that the abrupt dopamine denervation induced by standard 6-OHDA intoxication - weeks/months, in rats - is not fully appropriate for human PD. This may reflect the overwhelming role played by some compensatory mechanisms in the non-human models, at replicating most changes of the progressively developing disease in humans. By taking into account data collected in human and non-human primates, it is reasonable to speculate that some features of the standard model, if centered on the putative hyperactivity of $\mathrm{GPi} / \mathrm{SNr}$ as key factor underlying Parkinsonian OFF-state, are wrong.

At least three recent observations modify further our perception of BG functional circuitry. First, Brown and colleagues showed abundant evidence for the behavioral role of specific band frequencies (i.e., alpha rhythms for attention, beta for motor performance) linking cortex, STN and GPi. Even "acute" dopamine denervation (our TTX-mediated inactivation of the medial forebrain bundle, Galati et al., 2009, 2010) potentiated a synchronized low frequency discharge coupling STN-GPi to cortex. This pathological synergy between cortex and BG, in our hands, was suppressed by direct modulation of STN firing discharge by the gabamimetic muscimol and replicated by intra-GP injection of the D2-antagonist haloperidol. Overall, these findings reinforce the idea that fast motor commands are transferred through the hyper-direct pathway, alongside or in combination with changes of striatofugal networks.

Second, experiments performed with optogenetics supported the relevant role sub-served by cortico-STN pathway, if, as Gradinaru suggested, behaviorally effective high-frequency DBS was associated with a clear activation of axons impinging STN neurons.

Third, a renewed interest in non-cortical excitatory inputs to striatum (that is, thalamic outputs impinging on cholinergic interneurons) forces us to consider the possibility that the thalamoputaminal-pallidal loop plays a relevant role, as far as salient stimuli may perturb motor planning.

Overall, these considerations suggest that over-emphasis on fine details of circuitry may obscure a bigger picture.

The decrease of GABA concentration inside the motor thalamus (VA), during effective STN-DBS, reconciles with some aspects of the old model of BG function (Albin et al., 1989; DeLong, 1990). The GABA levels' reduction, in VA, would dis-inhibit VA firing activity and possibly influence its patterning; reduced bursting and/or suppressed oscillatory activity are the most likely results, as already inferred (Guo et al., 2008). The precise firing properties of human VA/VL during effective STN-DBS are unknown (but see Kempf et al., 2009) and we did not perform multiple recording traces in human PD VA on ethical grounds, since the latter is not a target of stimulation in PD (whilst appropriate in some pharmaco-resistant epileptic patients, Hodaie et al., 2006). However, previous studies may help the interpretation.

On one hand, GPi-DBS reduced VA/VL firing activity in normal monkeys (Anderson et al., 2003); on the other, Vitek described a mixed response in MPTP-intoxicated primates. Although the "lack of a significant reduction in the mean rate of discharge" was attributed to the early DBS-induced brisk excitation peak, there is no doubt that the "the most relevant changes due to effective DBS-STN was a dramatic modification of the PSTH, hence of patterning," characterized by "reduced bursting and more regular spike trains in thalamus" (Xu et al., 2008). Similarly, the Rubin and Terman (2004) model emphasized that STN-DBS, in order to be beneficial, induced clustered firing patterns in GPi, whose clear consequence is a regularization of VA discharge (more tonic, no pausing; see Figure 3).

As pointed out by Guo, STN-DBS altered Parkinsonian GPi activity in a way that may improve thalamocortical relay fidelity (Rubin and Terman, 2004; Guo et al., 2008). In this context, the reduced GABA levels that we observed would impede prolonged post-spiking after hyper-polarization, and favors a more regular pattern of neuronal activity (in striking contrast with the GABAergic and dopaminergic modulation of delta waves in reticular nuclei, i.e., Urbano et al., 2009).

The VA/VL nuclei complex represents the crucial link between BG outputs and specific cortical areas (Blandini et al., 2000; Obeso et al., 2008). Therefore, once VA-VL is modulated by STN-DBS (as a consequence of reduced GABA release), immediate changes in thalamocortical transmission would occur. Imaging studies have demonstrated a re-activation of fronto-temporo-parietal cortex at 


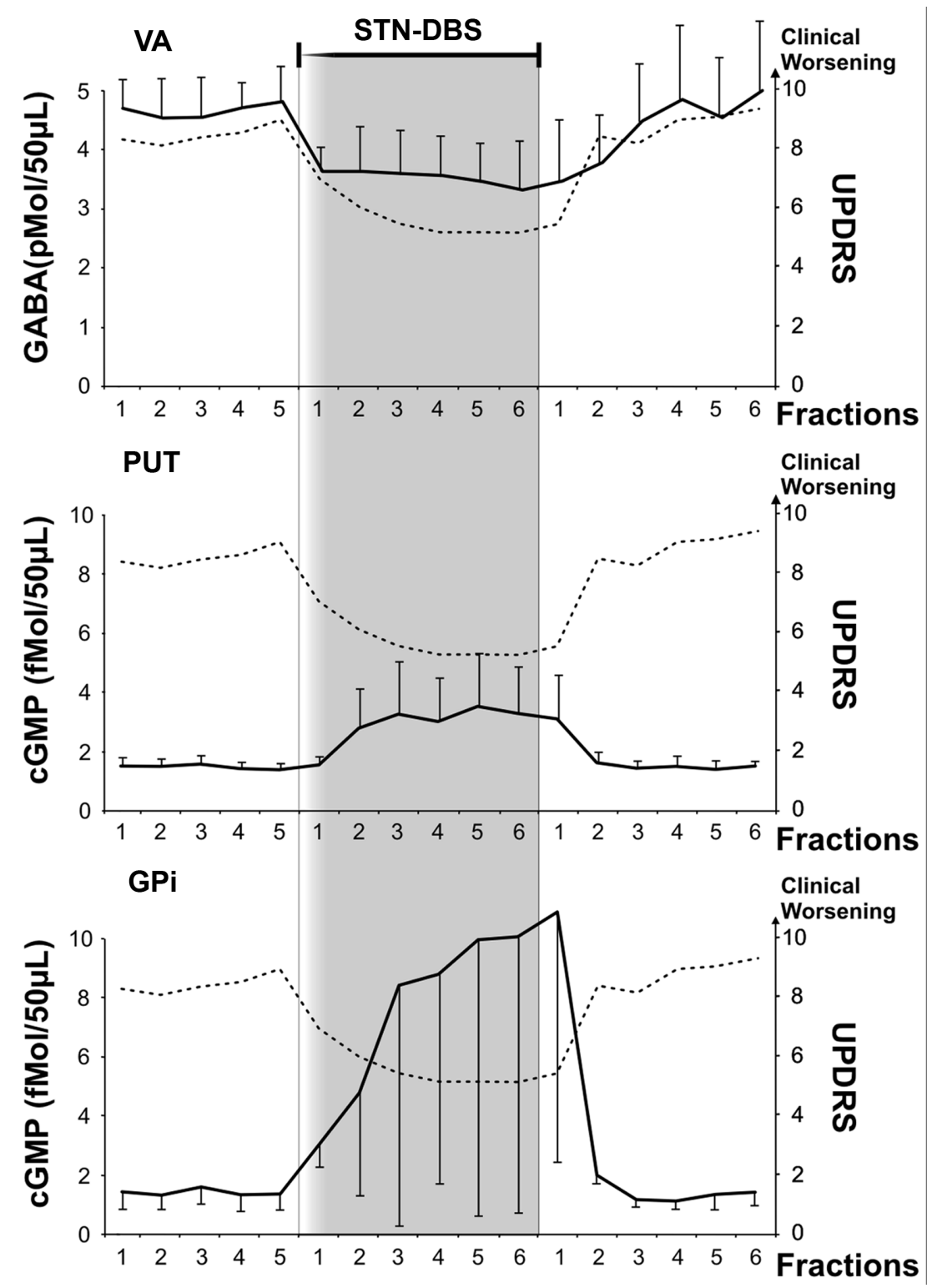

FIGURE 2 | Microdialysis data from VA, PUT and GPi and clinical evaluation before, during and after STN-DBS. Microdialysis probes were infused ( $5 \mu / / \mathrm{min})$ for stabilization (90 $\mathrm{min}$ ). During stabilization, the permanent stimulating electrode (Medtronic mod 3389) was implanted in ipsilateral STN. Following STN electrode insertion and probe stabilization, collection of 10-min fraction samples ( $50 \mu \mathrm{l}$ each) started. The first five fractions of each probe were utilized for basal evaluation either for GABA (VA probe, scale on the left $y$ axis in pmol/50 $\mu$ ) or for cGMP (PUT and GPi probes, scale on the left $y$ axis in $\mathrm{fmol} / 50 \mu \mathrm{l}$ ). Basal values are in the white area at the left of the shaded area. Values represent mean \pm SD. After Medtronic mod 3389 electrode implantation, monopolar STN-DBS was then switched on by a Medtronic external device Model 3625 at a rate between 130 and $160 \mathrm{~Hz}$, anodal pulse of 60-90 $\mu \mathrm{s}$, and voltage progressively increased by steps of $0.1 \mathrm{~V}$ up to the appearance of side effects (paresthesia or dystonia or myoclonus). Voltage was never above $3.0 \mathrm{~V}$. Voltage stimulation was then decreased by $10 \%$ or more to obtain disappearance of clinical side effects.
Clinical assessment started with microdialysis basal fraction collection. Clinical changes, contra lateral upper limb rigidity and akinesia were continuously assessed by an expert neurologist utilizing selected items of UPDRS (rigidity 0-4, finger tapping 0-4, hand movement 0-4; total $=0$ corresponds to "normal," 12 maximum score; scale on the right $y$ axis in each frame), while remaining blind to the stimulus intensity between 0 and $3 \mathrm{~V}$. The patient was unaware of the time of stimulus ON. In all the reported patients, an improvement of the sum of rigidity and akinesia of the upper contralateral arm of more than $30 \%$ was achieved without side effects within the first $10 \mathrm{~min}$ of stimulation at an intensity ranging between 2 and $3 \mathrm{~V}$, while the first fraction during stimulation was collected. STN-DBS intensity was kept constant thereafter and 10-min fractions were collected during $1 \mathrm{~h}$ of stimulation while clinical efficacy was continuously monitored to ensure persistence of the clinical effects. Clinical scores and biochemical parameters are reported every $10 \mathrm{~min}$. After $1 \mathrm{~h}, \mathrm{STN}-\mathrm{DBS}$ was discontinued and 10-min fractions were collected for one additional hour. rest after effective STN-DBS (Hilker et al., 2004, but see Hershey et al., 2003). Even in case STN-DBS apparently decreased rCBF in the motor cortex at rest - implying a reduction of abnormal overactivity in the motor system - this allowed selective cortical reactivation during active movement (Limousin et al., 1997; Payoux et al., 2004). 


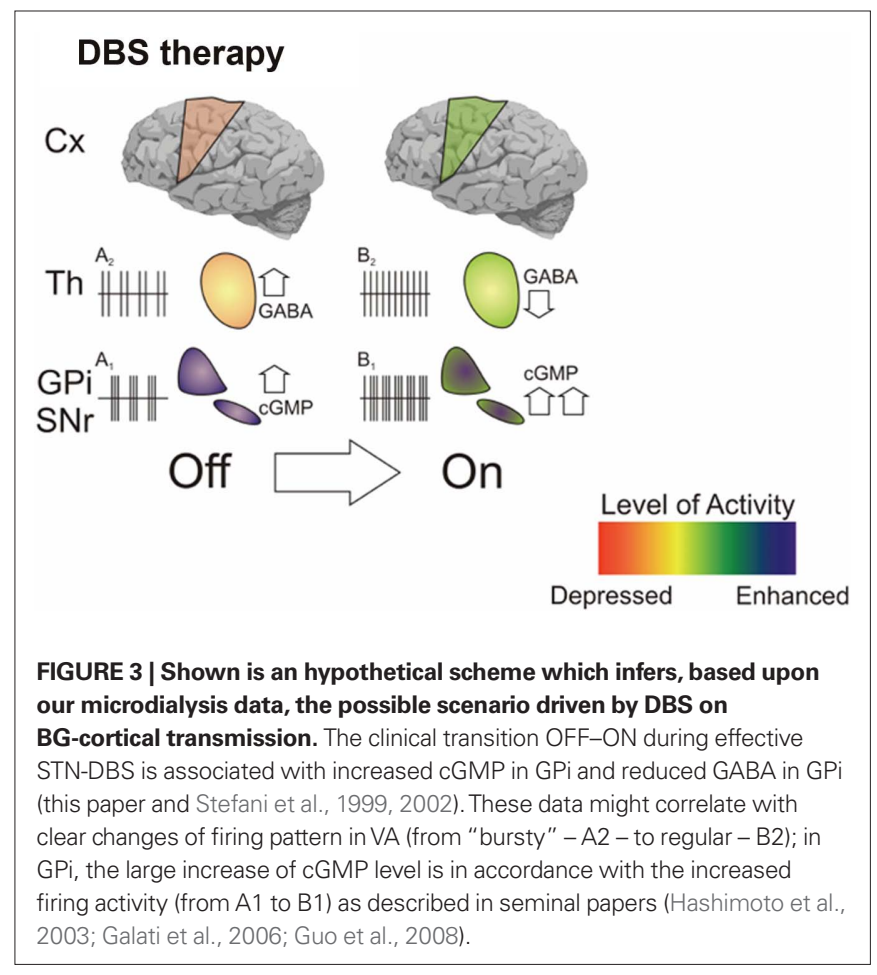

The two main effects induced by STN-DBS, as here described (increased cGMP in GPi and lower GABA in VA) seem in conflict each other, but do not necessarily correlate. STN-DBS indeed reverberate on multiple sites, including VTA and PPN extended area (Stefani et al., 2007), which in turn affect ascending pathways toward thalamus. In other words, the proteiform response to DBS on distant structures might overcome the strict boundaries of the direct/indirect pathway scheme.

Besides, the data concerning the DBS-mediated increase, inside PUT, of cGMP appear, in this context, as a further confirmation of the reinstalled physiological BG-cortex and cortico-BG transmission (otherwise impaired by PD OFF-state).

\section{FUNCTIONAL IMPLICATIONS}

We are showing a consistent, DBS-mediated GABA decline in the motor thalamus, which may associate with simultaneous increase of GPi and PUT cGMP.

A number of investigations from multiple disciplines are supporting the hypothesis that stimulation-induced modulation of pathological network activity represents the most likely mechanism of DBS (Brown, 2003). However, successful theories founded on the rebalancing of beta/gamma band frequencies should not discard, in agreement with recent conclusions by Johnson, Vitek, and McIntyre ("DBS improves Parkinsonian motor symptoms by inducing global changes in firing pattern and rate along the pallidothalamocortical sensorimotor circuit," Johnson et al., 2009), the central result of this report, that the motor thalamus is the critical station where efficacious re-modulation (electrical or chemical) of pathological outputs promotes good therapeutic outcomes.
So far, several considerations have limited the utilization of microdialysis in the surgery room; it represents a time-consuming approach, and ethical observations demand not to stress any patient if an immediate benefit is not perceived. Second, the "double-target" strategy has lost many fans, nowadays, making unlikely the simultaneous insertion of multiple electrodes in different BG stations. In other words, very few groups are still advocating these methods (but see Kilpatrick et al., 2010).

Yet, as we have tried to present in this paper, microdialysis had the profound merit to clarify the limitations of a dogmatic box and arrow cartoon scheme on BG circuitry, if merely intended as a simplistic balancing between overactive structures of the indirect pathway and facilitating structures governing the direct pathway. In this content, Figure 3 aims at presenting, albeit speculatively, findings derived from our last decade studies, again illustrating the importance to match functional recordings with alterations of endogenous transmitters.

In the next future, it is likely that newly developing approaches, centered on functional biochemistry, will be applied to PD patients. In particular, new electrochemical techniques, including fast-scan cyclic voltammetry (FSCV; Agnesi et al., 2009), should allow to detect changes that are happening on a second-to-second time scale (also feasible for chronically used implanted recordings, as would be required for DBS feedback).

As a matter of fact, the Wireless Instantaneous Neurochemical Concentration Sensor (WINCS) is currently a research device, whose resolution was established on pig brain, but it is designed for human use and is capable of in vivo FSCV plus amperometry, sampling at sub-second time resolution (Agnesi et al., 2009; Van Gompel et al., 2010). It promises, for instance, to assess if and to what extent effective STN-DBS is actually changing the tonic/phasic release of endogenous dopamine in human caudate/putamen (Covey and Garris, 2009; Van Gompel et al., 2010).

Overall, the implementation of these techniques will represent a real upgrade of our understanding of STN-DBS mechanisms of actions and, more critically, of our knowledge around the acute processes which rule clinical transitions in different extrapyramidal disorders.

\section{CONCLUSION}

Much recent literature has attempted to explain the mechanisms of action of DBS. Using optogenetic techniques, Gradinaru et al. (2009) have suggested that the more critical behaviorally related change promoted by high-frequency STN stimulation, in 6-OHDA rodents, is the direct activation of cortical axons to STN. This may stimulate new interest into DBS-mediated modulation of corticofugal pathways (i.e., the increase of putaminal cGMP that we have described), as inferred in early 2000s (Ashby et al., 2001).

On the other hand, in a brain slice preparation thalamostriatal axons exerted a marked modulation of firing in striatal cholinergic interneurons (in response to salient stimuli; Ding et al., 2010). This pathway would affect cortical input to medium spiny neuron (MSN), enhancing the postsynaptic responsiveness of striatopallidal MSNs, which strongly influence motor programming. This suggests that interpretation of the BG circuitry in terms of 
strict boundaries between direct and indirect pathways (limited to local, STN-pallidal interactions) is obsolete and must include a re-evaluation of thalamostriatal inputs.

That said, immediate transfer to the clinical practice is not easy. Cortical stimulation still retains some devotes, but is questionable as a first-line choice for treating movement disorders. Thalamic DBS regained interest through our small patient's series (Stefani et al., 2009), but is far from a consolidated target.

Overall, these considerations highlight once more the gap between rodent modeling and human disease. In humans, adaptive and compensatory mechanisms are far from well established, hence not easily replicable.

The microdialysis study presented here brings us back to solid data. We demonstrate that, whatever is the final modulation in cortex (1) GABA decline in the motor thalamus is central for the

\section{REFERENCES}

Agnesi, F., Tye, S. J., Bledsoe, J. M., Griessenauer, C. J., Kimble, C. J., Sieck, G. C., Bennet, K. E., Garris, P.A., Blaha, C. D., and Lee, K. H. (2009). Wireless instantaneous neurotransmitter concentration system-based amperometric detection of dopamine, adenosine, and glutamate for intraoperative neurochemical monitoring. J. Neurosurg. 111, 701-711.

Albin, R. L., Young, A. B., and Penney, J. B. (1989). The functional anatomy of basal ganglia disorders. Trends Neurosci. 5, 366-375.

Anderson, M.E., Postupna, N., and Ruffo, M. (2003). Effects of high-frequency stimulation in the internal globus pallidus on the activity of thalamic neurons in the awake monkey. $J$. Neurophysiol. 89, 1150-1160.

Ashby, P., Paradiso, G., Saint-Cyr, J. A., Chen, R., Lang, A. E., and Lozano, A. M. (2001). Potentials recorded at the scalp by stimulation near the human subthalamic nucleus. Clin. Neurophysiol. 112, 431-437.

Benazzouz,A., Gao, D.M., Ni,Z.G., Piallat, B., Bouali-Benazzouz, R., and Benabid, A. L. (2000). Effect of high-frequency stimulation of the subthalamic nucleus on the neuronal activities of the substantia nigra pars reticulata and ventrolateral nucleus of the thalamus in the rat. Neuroscience 99, 289-295.

Blandini, F., Nappi, G., Tassorelli, C., and Martignoni, E. (2000). Functional changes of the basal ganglia circuitry in Parkinson's disease. Prog. Neurobiol. 62, 63-88.

Brown, P. (2003). Oscillatory nature of human basal ganglia activity: relationship to the pathophysiology of Parkinson's disease. Mov. Disord. 18, 357-363.

Covey, D. P., and Garris, P.A. (2009). Using fast-scan cyclic voltammetry to evaluate striatal dopamine release elicited by subthalamic nucleus stimulation. Conf. Proc. IEEE Eng. Med. Biol. Soc. 3306-3309.

DeLong, M. R. (1990). Primate models of movement disorders of basal ganglia origin. Trends Neurosci. 13, 281-285.

Ding, J. B., Guzman, J. N., Peterson, J. D., Goldberg, J. A., and Surmeier, D. J. (2010). Thalamic gating of corticostriatal signaling by cholinergic interneurons. Neuron 67, 294-307.

Fedele, E., Mazzone, P., and Stefani, A. (2001). Microdialysis in Parkinsonian patient basal ganglia: acute apomorphine-induced clinical and electrophysiological effects not paralleled by changes in the release of neuroactive amino acids. Exp. Neurol. 167, 356-365.

Fedele, E., and Raiteri, M. (1999). In vivo studies of the cerebral glutamate receptor/NO/cGMP pathway. Prog. Neurobiol. 58, 89-120.

Filali, M., Hutchison, W. D., Palter, V. N., Lozano, A. M., and Dostrovsky, J. O. (2004). Stimulation-induced inhibition of neuronal firing in human subthalamic nucleus. Exp. Brain Res. 156, 274-281.

Follett, K. A., Weaver, F. M., Stern, M., Hur, K., Harris, C. L., Luo, P., Marks, W. J., Rothlind, J., Sagher, O., Moy, C., Pahwa, R., Burchiel, K., Hogarth, P., Lai, E. C., Duda, J. E., Holloway, K., Samii, A., Horn, S., Bronstein, J. M., Stoner, G., Starr, P. A., Simpson, R., Baltuch, G., De Salles, A., Huang, G.D., and Reda, D. J. (2010). Pallidal versus subthalamic deep-brain stimulation for Parkinson's disease. N. Engl. J. Med. 362, 2077-2091.

Galati, S., D’Angelo, V., Olivola, E., Marzetti, F., Di Giovanni, G., Stanzione, P., and Stefani, A. (2010). Acute inactivation of the medial forebrain bundle imposes oscillations in the SNr: a challenge for the 6-OHDA model? Exp. Neurol. 225, 294-301.

clinical amelioration but (2) that the latter may associate (under DBS) or not (under LD, Stefani et al., 2011) with changes of GPi cGMP (a marker of STN-pallidal excitability). In other words, the modulation of VA GABA, clearly relevant for clinical score, occurs despites on-going changes of pallidal discharge.

As a parsimonious explanation, during LD-mediated clinical amelioration, pathways other than the pallidothalamic, but still affecting VA, indeed contribute to the rebalancing of the thalamocortical output. During STN-DBS, the reduction in VA GABA content derives from complex mechanisms, including selective activation of small pallidothalamic fibers or direct modulation of pallidal neuropil.

\section{ACKNOWLEDGMENTS}

This work has been supported by Ministero della Salute Grants to Paolo Stanzione and Alessandro Stefani.

Galati, S., Mazzone, P., Fedele,E., Pisani,A., Peppe, A., and Pierantozzi, M. (2006). Biochemical and electrophysiological changes of substantia nigra pars reticulata driven by subthalamic stimulation in patients with Parkinson's disease. Eur. J. Neurosci. 23, 2923-2928.

Galati, S., Stanzione, P., D’Angelo, V. Fedele, E., Marzetti, F., Sancesario, G., Procopio, T., and Stefani, A. (2009). The pharmacological blockade of medial forebrain bundle induces an acute pathological synchronization of the cortico-subthalamic nucleusglobus pallidus pathway. J. Physiol. 587, 4405-4423.

Gradinaru, V., Mogri, M., Thompson K. R., Henderson, J. M., and Deisseroth, K. (2009). Optical deconstruction of Parkinsonian neural circuitry. Science 324, 354-359.

Guo, Y., Rubin, J. E., McIntyre, C. C. Vietk, J. L., and Terman, D. (2008). Thalamocortical relay fidelity varies across subthalamic nucleus deep brain stimulation protocols in a data-driven computational model. J. Neurophysiol. 99, 1477-1492.

Hahn, P. J., Russo, G. S., Hashimoto, T. Miocinovic, S., Xu, W., McIntyre, C. C., and Vitek, J. L. (2008). Pallidal burst activity during therapeutic deep brain stimulation. Exp. Neurol. 211, 243-251.

Hashimoto, T., Elder, C. M., Okun, M. S., Patrick, S. K., and Vitek, J. L. (2003). Stimulation of the subthalamic nucleus changes the firing pattern of pallidal neurons. J. Neurosci. 23, 1916-1923.

Hershey, T., Revilla, F. J., Wernle, A. R., McGee-Minnich, L., Antenor, J. V., and Videen, T. O. (2003). Cortical and subcortical blood flow effects of subthalamic nucleus stimulation in PD. Neurology 61, 816-821.

Hilker, R., Voges, J., Weisenbach, S., Kalbe, E., Burghaus, L., and Ghaemi, M.
(2004). Subthalamic nucleus stimulation restores glucose metabolism in associative and limbic cortices and in cerebellum: evidence from a FDG-PET study in advanced Parkinson's disease. J. Cereb. Blood Flow Metab. 24, 7-16.

Hodaie, M., Cordella, R., Lozano, A. M., Wennberg, R., and Dostrovsky, J. O. (2006). Bursting activity of neurons in the human anterior thalamic nucleus. Brain Res. 1115, 1-8.

Johnson, M. D., Vitek, J. L., and McIntyre, C. C. (2009). Pallidal stimulation that improves Parkinsonian motor symptoms also modulates neuronal firing patterns in primary motor cortex in the MPTP-treated monkey. Exp. Neurol. 219, 359-362.

Kempf, F., Brücke, C., Salih, F., Trottenberg, T., Kupsch, A., and Schneider GH. (2009). Gamma activity and reactivity in human thalamic local field potentials. Eur. J. Neurosci. 29, 943-953.

Kilpatrick, M., Church, E., Danish, S., Stiefel, M., Jaggi, J., and Halpern, C. (2010). Intracerebral microdialysis during deep brain stimulation surgery. J. Neurosci. Methods 190, 106-111.

Limousin, P., Greene, J., Pollak, P., Rothwell, J., Benabid, A. L., and Frackowiak, R. (1997). Changes in cerebral activity pattern due to subthalamic nucleus or internal pallidum stimulation in Parkinson's disease. Ann. Neurol. 42, 283-291.

Obeso, J. A., Rodríguez-Oroz, M. C., Benitez-Temino, B., Blesa, F. J., Guridi, J., and Marin, C. (2008). Functional organization of the basal ganglia: therapeutic implications for Parkinson's disease. Mov. Disord. 23, S548-S559.

Payoux, P., Remy, P., Damier, P., Miloudi, M., Loubinoux, I., Pidoux, B., Gaura, V., Rascol, O., Samson, Y., and Agid, Y. (2004). Subthalamic nucleus stimulation reduces abnormal motor cortical overactivity in Parkinson disease. Arch. Neurol. 61, 1307-1313. 
Peppe, A., Pierantozzi, M., Bassi, A., Altibrandi, M. G., Brusa, L., Stefani, A., Stanzione, P., and Mazzone, P. (2004). Stimulation of the subthalamic nucleus compared with the globus pallidus internus in patients with Parkinson disease. J. Neurosurg. 101, 195-200.

Rubin,J.E., and Terman,D. (2004). High frequency stimulation of the subthalamic nucleus eliminates pathological thalamic rhythmicity in a computational model. J. Comput. Neurosci. 16,211-235.

Shi, L. H., Luo, F., Woodward, D. J., and Chang, J. Y. (2006). Basal ganglia neural responses during behaviorally effective deep brain stimulation of the subthalamic nucleus in rats performing a treadmill locomotion test. Synapse 59, 445-457.

Stefani, A., Bassi, A., Mazzone, P., Pierantozzi, M., Gattoni, G.,Altibrandi, M. G., Giacomini, P., Peppe, A., Bernardi, G., and Stanzione, P. (2002). Subdyskinetic apomorphine responses in globus pallidus and subthalamus of Parkinsonian patients: lack of clear evidence for the 'indirect pathway'. Clin. Neurophysiol. 113, 91-100.

Stefani, A., Fedele, E., Galati, S., Pepicelli, O., Frasca, S., and Pierantozzi, M. (2005). Subthalamic stimulation activates internal pallidus: evidence from cGMP microdialysis in PD patients. Ann. Neurol. 57, 448-452.
Stefani, A., Fedele, E., Galati, S., Raiteri, M., Pepicelli, O., and Brusa, L. (2006). Deep brain stimulation in Parkinson's disease patients: biochemical evidence. J. Neural Transm. 70, S401-S408.

Stefani, A., Fedele, E., Vitek, J., Pierantozzi, M., Galati, S., Marzetti, F., Peppe, A., Bernardi, G., and Stanzione, P. (2011). The clinical efficacy of L-dopa and STN-DBS share a common marker: reduced GABA content in the motor thalamus. Cell Death Dis. (in press).

Stefani, A., Lozano, A. M., Peppe, A., Stanzione, P., Galati, S., and Tropepi, D. (2007). Bilateral deep brain stimulation of the pedunculopontine and subthalamic nuclei in severe Parkinson's disease. Brain 130, 1596-1607.

Stefani, A., Mazzone, P., Bassi, A., Bernardi, G., Altibrandi, M. G., Peppe, A., Pierantozzi, M., and Stanzione, P. (1999). Electrophysiological and clinical desensitization to apomorphine administration in Parkinsonian patients undergoing stereotaxic neurosurgery. Exp. Neurol. 156, 209-213.

Stefani, A., Peppe, A., Pierantozzi, M., Galati, S., Moschella, V., and Stanzione, P. (2009). Multi-target strategy for Parkinsonian patients: the role of deep brain stimulation in the centromedian-parafascicularis complex. Brain Res. Bull. 78, 113-118.

Stefani,A.,Stanzione, P., Bassi,A., Mazzone, P., Vangelista, T., and Bernardi, G.
(1997). Effects of increasing doses of apomorphine during stereotaxic neurosurgery in Parkinson's disease: clinical score and internal globus pallidus activity. J. Neural. Transm. 104, 895-904.

Urbano, F. J., Bisagno, V., Wikinski, S. I., Uchitel, O. D., and Llinás, R. R. (2009). Cocaine acute "binge" administration results in altered thalamocortical interactions in mice. Biol. Psychiatry 66, 769-776.

Van Gompel, J. J., Chang, S. Y., Goerss, S. J., Kim, I. Y., Kimble, C., Bennet, K. E. and Lee, K. H. (2010). Development of intraoperative electrochemical detection: wireless instantaneous neurochemical concentration sensor for deep brain stimulation feedback. Neurosurg. Focus 29, E6.

Windels, F., Bruet, N., Poupard, A., Feuerstein, C., Bertrand, A., and Savasta, M. (2003). Influence of the frequency parameter on extracellular glutamate and gamma-aminobutyric acid in substantia nigra and globus pallidus during electrical stimulation of subthalamic nucleus in rats. J. Neurosci. Res. 72, 259-267.

Windels, F., Carcenac, C., Poupard, A., and Savasta, M. (2005). Pallidal origin of GABA release within the substantia nigra pars reticulata during high-frequency stimulation of the subthalamic nucleus. J. Neurosci. 25, 5079-5086.
Xu, W., Russo, G.S., Hashimoto, T., Zhang, J., and Vitek, J. L. (2008). Subthalamic nucleus stimulation modulates thalamic neuronal activity. J. Neurosci. 28, 11916-11924.

Conflict of Interest Statement: The authors declare that the research was conducted in the absence of any commercial or financial relationships that could be construed as a potential conflict of interest.

Received: 30 December 2010; paper pending published: 02 February 2011; accepted: 22 March 2011; published online: 05 April 2011.

Citation: Stefani A, Fedele E, Pierantozzi M, Galati S, Marzetti F, Peppe A, Pastore FS, Bernardi G and Stanzione P (2011) Reduced GABA content in the motor thalamus during effective deep brain stimulation of the subthalamic nucleus. Front. Syst. Neurosci. 5:17. doi: 10.3389/ fnsys.2011.00017

Copyright (C) 2011 Stefani, Fedele, Pierantozzi, Galati, Marzetti, Peppe, Pastore, Bernardi and Stanzione. This is an open-access article subject to a nonexclusive license between the authors and Frontiers Media SA, which permits use, distribution and reproduction in other forums, provided the original authors and source are credited and other Frontiers conditions are complied with. 